\title{
INVARIANT MEASURES AND STIFFNESS FOR NON ABELIAN GROUPS OF TORAL AUTOMORPHISMS
}

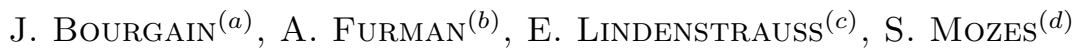 \\ (a) Institute for Advanced Study, Princeton, NJ 08540 \\ (b) University of Illinois at Chicago, Chicago, IL 60607 \\ (c) Princeton University, Princeton, NJ 08544 \\ (d) The Hebrew University, 91904 Jerusalem, Israel
}

\begin{abstract}
Let $\Gamma$ be a non-elementary subgroup of $\mathrm{SL}_{2}(\mathbb{Z})$. If $\mu$ is a probability measure on $\mathbb{T}^{2}$ which is $\Gamma$-invariant, then $\mu$ is a convex combination of the Haar measure and an atomic probability measure supported by rational points. The same conclusion holds under the weaker assumption that $\mu$ is $\nu$-stationary, i.e. $\mu=\nu * \mu$, where $\nu$ is a finitely supported probability measure on $\Gamma$ whose support $\operatorname{supp}(\nu)$ generates $\Gamma$. The approach works more generally for $\Gamma<\mathrm{SL}_{d}(\mathbb{Z})$.
\end{abstract}

RESUmE. Soit $\Gamma$ un sous-groupe non-élementaire du groupe $\mathrm{SL}_{2}(\mathbb{Z})$. Soit $\mu$ une measure de probabilité $\Gamma$-invariante sur le tore $\mathbb{T}^{2}$. On démontre que $\mu$ est une moyenne de la mesure de Haar et une probabilité discrète portée par des points rationnels. La même conclusion reste vrai sous l'hypothèse que $\mu$ est $\nu$-stationnaire, done $\mu=\nu * \mu$, où $\nu$ est une probabilité sur $\Gamma$ à support fini et engendrant $\Gamma$. L'approche se généralise aux sous-groupes $\Gamma$ de $\mathrm{SL}_{d}(\mathbb{Z})$. 


\section{Version française abrégée}

Nous considérons l'action de $\mathrm{SL}_{2}(\mathbb{Z})$ sur le tore $\mathbb{T}^{2}$. Soit $\Gamma$ un sous-groupe nonélémentaire du $\mathrm{SL}_{2}(\mathbb{Z})$. Soit $\mu$ une measure sur $\mathbb{T}^{2}$ que nous supposons $\Gamma$-invariante, ou, moins restrictivement, que $\mu$ est $\nu$-stationnaire pour une probabilité $\nu$ sur $\Gamma$ à support fini et tel que $\langle\operatorname{supp}(\nu)\rangle=\Gamma$. Nous démontrons que si $\mu$ n'est pas un multiple de la mesure de Haar sur $\mathbb{T}^{2}$, alors $\mu$ a une composante discrète. La méthode comporte plusieurs étapes est des techniques d'analyse harmonique y jouent un rôle essentiel. Supposons la transformé de Fourier $\hat{\mu}(b) \neq 0$ pour un élément $b \in \mathbb{Z}^{2} \backslash\{0\}$. Le point de départ consiste à étudier l'ensemble $\Lambda_{c}=\left\{n \in \mathbb{Z}^{2} ;|\hat{\mu}(n)|>c\right\}(c>0$ approprié) et de montrer que $\Lambda_{c}$ est 'riche', en un certain sense d'entropie métrique. On utilise ici divers arguments d'amplification et un résultat d'équirépartition pour convolutions multiplicatives sur $\mathbb{R}$, qui repose sur le théorème 'somme-produit' obtenu dans [B] et [BG]. Ensuite on déduit de la structure de $\Lambda_{c}$ des propriétés de 'porosité' pour le support de $\mu$ et finalement une composante discrète.

In this Note we present some new dichotomies for invariant and stationary measures $\mu$ on $\mathbb{T}^{2}$ under the action of $\mathrm{SL}_{2}(\mathbb{Z})$-subgroups.

Theorem A. If $\mu$ is invariant under the action of a non-elementary subgroup $\Gamma$ of $\mathrm{SL}_{2}(\mathbb{Z})$, then $\mu$ is a linear combination of Haar measure on $\mathbb{T}^{2}$ and an atomic measure supported by rational points.

Theorem B. The same conclusion holds if we assume $\mu$ is $\nu$-stationary, i.e. $\mu=$ $\nu * \mu=\sum_{g \in \Gamma} \nu(g) g_{*} \mu$, with $\nu$ a finitely supported probability measure on $\mathrm{SL}_{2}(\mathbb{Z})$ such that $\Gamma=\langle\operatorname{supp}(\nu)\rangle$ is a non-elementary subgroup.

Theorem C. If for a point $\theta \in \mathbb{T}^{2}$ the measure $\eta_{n}=\nu^{(n)} * \delta_{\theta}$ has Fourier coefficient $\left|\hat{\eta}_{n}(b)\right|>\delta$ for some $b \in \mathbb{Z}^{2} \backslash\{0\}$, then $\theta$ admits a rational approximation

$$
\left\|\theta-\frac{a}{q}\right\|<e^{-c n} \quad \text { for some } q<\left(\frac{\|b\|}{\delta}\right)^{C}
$$

with $c, C>0$ depending on $\nu$.

Theorem C answers the question of equidistribution, posed by Y. Guivarc'h [G]. 
Theorem D. Unless $\theta \in \mathbb{T}^{2}$ is rational, $\nu^{(n)} * \delta_{\theta}$ tend weak ${ }^{*}$ to Lebesgue measure as $n \rightarrow \infty$.

Comments. (1) The results extend to $\mathrm{SL}_{d}(\mathbb{Z})$, assuming that $\operatorname{supp}(\nu)$ generates a Zariski dense subgroup in $\mathrm{SL}_{d}(\mathbb{R})$ or, more generally, assuming that the smallest algebraic subgroup $H_{\nu} \subset \mathrm{SL}_{d}(\mathbb{R})$ supporting $\nu$, is strongly irreducible (leaves invariant no finite union of $\mathbb{R}^{d}$-hyperplanes) and contains a proximal element. Under these conditions the top exponent is simple (see $[\mathrm{G}-\mathrm{M}]$ ).

(2) $\nu$-stationary measures play an important role in the theory of boundaries of groups, and were systematically used by H. Furstenberg and others in many works. In his paper [F2] H. Furstenberg explores the relationship between $\nu$-stationary measures and $\Gamma$-invariant measures, where $\nu$ is a probability measure on $\Gamma$ whose support generates $\Gamma$. For a general action of $\Gamma$ on a space $X$ there is a big difference between the two concepts: indeed, if $X$ is compact $\nu$-stationary measures always exist but there may well be no $\Gamma$-invariant probability measure whatsoever. In [F2] Furstenberg introduces the notion of stiff actions: an action of a group $\Gamma$ on a space $X$ is said to be $\nu$-stiff if every $\nu$-stationary measure is in fact $\Gamma$-invariant, and proves stiffness for the action of $\Gamma=\mathrm{SL}_{d}(\mathbb{Z})$ on $\mathbb{T}^{d}$ where $\nu$ is a (very) carefully chosen probability measure on $\mathrm{SL}_{d}(\mathbb{Z})$.

Furstenberg conjectures that this action is stiff for any $\nu$ whose support generates $\operatorname{SL}(d, \mathbb{Z})$. Theorem $\mathrm{B}$ and its extension to $d>2$ establish in particular this conjecture. Moreover, in conjunction with strong approximation results such as those in $[\mathrm{We}],[\mathrm{P}]$, our results imply that the action is "superstiff", in the sense that if $\langle\operatorname{supp}(\nu)\rangle$ is Zariski dense in $\mathrm{SL}_{d}(\mathbb{R})$, any $\nu$-stationary measure on $\mathbb{T}^{d}$ is invariant under a finite index subgroup of $\mathrm{SL}_{d}(\mathbb{Z})$ (depending only on $\operatorname{supp}(\nu)$ ).

(3) Theorem A may be viewed as a non-Abelian analogue of the wellknown $\times 2, \times 3$ invariant measure problem on the circle $\mathbb{T}$. Thus the conjecture states that if $\mu \in$ $M(\mathbb{T})$ satisfies $\hat{\mu}(n)=\hat{\mu}(2 n)=\hat{\mu}(3 n)$ for all $n \in \mathbb{Z}$, then $\mu$ is a combination of Haar and discrete measures. It is known that if we assume moreover that $\mu$ has positive entropy, then $\mu$ is Haar (see $[\mathrm{R}]$ and $[\mathrm{Ka}-\mathrm{K}],[\mathrm{K}-\mathrm{S}]$, [E-L] for the generalization to $\mathbb{Z}^{d}$-actions on tori). However, in the context of $\times 2, \times 3$ problem, or its toral analogues, statements such as Theorem D do not hold. 
(4) We also recall that there are (Abelian and non-Abelian) counterparts for orbit closures. In the Abelian case, these are the dichotomy results of H. Furstenberg $[\mathrm{F} 1]$ and D. Berend [Be]. The non-Abelian problem for $\Gamma$-orbits, $\Gamma \subset \mathrm{SL}_{d}(\mathbb{Z})$ a semigroup action on $\mathbb{T}^{d}$, appears for example in G. Margulis list of open problems $[\mathrm{M}]$. Contributions here include the work of A. Starkov [St] (for $\Gamma$ a strongly irreducible subgroup of $\mathrm{SL}_{d}(\mathbb{Z})$ ), R. Muchnik [M1], [M2] ( $\Gamma$ a Zariski dense semigroup) and Guivarc'h-Starkov [G-S].

Next, we give a brief overview of the proof of Theorem B. The proof of Theorem C (which implies D, B and A) uses the same ingredients - see comments at the end. There are several distinct steps in the proofs which we summarize.

Assume $\mu$ to be a $\nu$-stationary probability measure on $\mathbb{T}^{2}$ different from the Haar measure. Thus

$$
\hat{\mu}(b) \neq 0 \quad \text { for some } \quad b \in \mathbb{Z}^{2} \backslash\{0\}
$$

and hence

$$
\sum_{g}\left|\hat{\mu}\left(g^{t}(b)\right)\right| \cdot \nu^{(r)}(g) \geq|\hat{\mu}(b)|=\alpha>0
$$

for any convolution power $\nu^{(r)}$ of $\nu$. It is clear from (2) that $\mu$ has many large Fourier coefficients; in fact, there is $\delta>0$ such that

$$
\mid\left\{n \in \mathbb{Z}^{2}:\|n\| \leq N \text { and }|\hat{\mu}(n)|>\frac{1}{2} \alpha\right\} \mid>N^{\delta}
$$

for all sufficiently large $N$. However, unless $\delta$ is sufficiently close to 2 , we need a more structured set of large Fourier coefficients. This is achieved in

Step 1. (amplification).

Lemma 1. There are positive constants $\beta>0$ and $\kappa>0$ such that for all sufficiently large $N \in \mathbb{Z}_{+}$, there is a set $\mathcal{F} \subset \mathbb{Z}^{2} \cap B(0, N)$ with the following properties

(a) $|\hat{\mu}(k)|>\beta$ for $k \in \mathcal{F}$.

(b) $\left\|k-k^{\prime}\right\|>N^{1-\kappa}$ if $k \neq k^{\prime}$ in $\mathcal{F}$.

(c) $|\mathcal{F}|>\beta N^{2 \kappa}$.

Our proof of Lemma 1 is rather involved. It is obtained by combining the following ingredients. 
Denote by $\delta(\bar{x}, \bar{y})$ the angular distance on the projective space $P\left(\mathbb{R}^{2}\right)$. The following statement is obtained by combining Proposition 4.1 (p. 161) and Theorem 2.5 (p. 106) from [B-L].

Proposition 2 (small ball estimate). There is a uniform estimate for $\bar{x}, \bar{y} \in P\left(\mathbb{R}^{2}\right)$

$$
\nu^{(n)}\{g: \delta(g \bar{x}, \bar{y})<\varepsilon\}<C\left(\varepsilon^{\alpha}+e^{-c n}\right)
$$

for some $\alpha, c, C>0$.

We also use the large deviation estimate for the Lyapounov exponent $\gamma$ (Theorem 6.2 , p. 131 in $[\mathrm{B}-\mathrm{L}])$, which gives:

Proposition 3. Uniformly in $x,\|x\|=1$ :

$$
\nu^{(n)}\left\{g:\left|\frac{1}{n} \log \|g x\|-\gamma\right|>\frac{\gamma}{10}\right\}<C e^{-c n}
$$

The combinatorial information that can be extracted from Proposition 2 on the set of large Fourier coefficients is amplified using the following general statement on mixed multiplicative and additive convolution on $\mathbb{R}$ (which may be of independent interest).

Proposition 4. Given $\theta>0, C>1$, there are $s \in \mathbb{Z}_{+}$and $C^{\prime}>1$ such that the following holds.

Let $\delta>0$ and $\eta$ a probability measure on $\left[\frac{1}{2}, 1\right]$ satisfying

$$
\max _{a} \eta(B(a, \rho))<C \rho^{\theta} \quad \text { for } \quad \delta<\rho<1 .
$$

Consider the image measure $\nu$ of $\eta \otimes \cdots \otimes \eta\left(s^{2}\right.$-fold $)$ under the map

$$
\left(x_{1}, \ldots, x_{s^{2}}\right) \mapsto\left(x_{1} \ldots x_{s}\right)+\left(x_{s+1} \ldots x_{2 s}\right)+\cdots+\left(x_{s^{2}-s+1} \ldots x_{s^{2}}\right) .
$$

Then

$$
\max _{a} \nu(B(a, \rho))<C^{\prime} \rho \quad \text { for } \quad \delta<\rho<1
$$

where here $B(a, \rho)=[a-\rho, a+\rho]$.

Proposition 3 is deduced from a set-theoretical statement, which is the 'discretized ring conjecture' (in the sense of $[\mathrm{K}-\mathrm{T}]$ ); see $[\mathrm{B}]$, [B-G].

Returning to Lemma 1, there is the following implication on the support of $\mu$.

Step 2. (porosity property). 
Using elementary harmonic analysis, one shows the following general.

Lemma 5. Let $\mu$ be a probability measure on $\mathbb{T}^{d}, d \geq 1$. Fix $\kappa_{1}, \kappa_{2}>0$.

Let $N \gg M$ be large integers and assume

$$
\mathcal{N}\left(\left[|\hat{\mu}|>\kappa_{1}\right] \cap B(0, N) ; M\right)>\kappa_{2}\left(\frac{N}{M}\right)^{d}
$$

where for $A \subset \mathbb{Z}^{d}$ and $R>1, \mathcal{N}(A ; R)$ denotes the smallest number of balls of radius $R$ needed to cover $A$.

Then there are points $x_{1}, \ldots, x_{\beta} \in \mathbb{T}^{d}$ such that

$$
\begin{aligned}
& \left\|x_{\alpha}-x_{\alpha^{\prime}}\right\|>\frac{1}{M} \quad \text { for } \quad \alpha \neq \alpha^{\prime} \\
& \sum_{\alpha} \mu\left(B\left(x_{\alpha}, \frac{1}{N}\right)\right)>\rho\left(\kappa_{1}, \kappa_{2}\right)>0 .
\end{aligned}
$$

Combined with Lemma 1 ( $d=2$ and taking $\left.\kappa_{1}=\beta=\kappa_{2}, M=N^{1-\kappa}\right)$, we obtain therefore

Lemma 6. For all $N$ large enough, there are points $x_{1}, \ldots, x_{\beta} \in \mathbb{T}^{2}$ such that $\left\|x_{\alpha}-x_{\alpha^{\prime}}\right\|>\frac{1}{N^{1-\kappa}}$ for $\alpha \neq \alpha^{\prime}$ and

$$
\sum_{\alpha} \mu\left(B\left(x_{\alpha}, \frac{1}{N}\right)\right)>\rho .
$$

Our next aim is to improve the porosity property obtained in Lemma 4 by decreasing the radius of the balls.

Step 3. (bootstrap).

Starting from the statement in Lemma 4 and using the group action, we prove

Lemma 7. For any fixed number $C_{0}$, there is a collection of points $\left\{z_{\alpha}\right\} \in \mathbb{T}^{2}$ such that

$$
\left\|z_{\alpha}-z_{\alpha^{\prime}}\right\|>\frac{1}{2 N^{1-\kappa}}>\frac{1}{N} \quad \text { for } \quad \alpha \neq \alpha^{\prime}
$$

and

$$
\sum_{\alpha} \mu\left(B\left(z_{\alpha}, \frac{1}{N^{C_{0}}}\right)\right)>\rho\left(C_{0}\right)>0 .
$$


The statement follows from a simple iterative construction. Under the action of $\mathrm{SL}_{2}(\mathbb{Z})$-elements, the balls become elongated ellipses and intersecting different families leads to sets of smaller diameter.

Step 4. (rational approximation).

Assume

$$
\mu(B(x, \varepsilon))>\varepsilon^{\tau}
$$

where $\varepsilon>0$ is small and $\tau>0$ a fixed exponent.

Take $n \sim\left(\frac{1}{\varepsilon}\right)^{1 / 2}$ and make a diophantine approximation

$$
\left|x_{1}-\frac{a_{1}}{q}\right|<\frac{1}{q \sqrt{n}}, \quad\left|x_{2}-\frac{a_{2}}{q}\right|<\frac{1}{q \sqrt{n}}
$$

where $1 \leq q \leq n$ and $\operatorname{gcd}\left(a_{1}, a_{2}, q\right)=1$. It follows from (3), (4) that

$$
\mu\left(B\left(\frac{a}{q}, \frac{2}{q \sqrt{n}}\right)\right)>\varepsilon^{\tau}
$$

and the $\nu$-stationarity of $\mu$ implies for any $r \in \mathbb{Z}_{+}$

$$
\sum_{g} \mu\left(B\left(\frac{g(a)}{q}, \frac{2\|g\|}{q \sqrt{n}}\right)\right) \cdot \nu^{(r)}(g)>\varepsilon^{\tau} .
$$

Take $r \sim \log n$ as to ensure that $\|g\|<n^{1 / 3}$ if $g \in \operatorname{supp} \nu^{(r)}$. It follows then from (5) and our choice of $r$ that

$$
\varepsilon^{\tau} \leq \sum_{b \in \mathbb{Z}_{q}^{2}} \mu\left(B\left(\frac{b}{q}, \frac{1}{2 q}\right)\right) \cdot \nu^{(r)}(\{g \mid g a \equiv b(\bmod q)\}) .
$$

A spectral gap of the form $\left\|\nu^{(r)}\right\| \leq q^{-\omega_{1}}, r \geq \log q$, on $\ell^{2}\left(\mathbb{Z}_{q}^{2}\right) \ominus \mathbb{C}$ with some fixed $\omega_{1}>0$ depending only on $\nu$, yields the estimate

$$
\begin{gathered}
\max _{b \in \mathbb{Z}_{q}^{2}} \nu^{(r)}(\{g \mid g a \equiv b(\bmod q)\})<q^{-\omega} . \\
q<\left(\frac{1}{\varepsilon}\right)^{\tau / \omega} .
\end{gathered}
$$

Recalling the conclusion of Lemma 5, the exponent $\tau$ in (3) may be taken to be an arbitrary small fixed positive number. In particular, we may ensure that in (6), $q<Q(\varepsilon)<\left(\frac{1}{\varepsilon}\right)^{\frac{1}{20}}$. Thus we proved that there is $\rho_{1}>0$ such that for all $\varepsilon>0$ small enough

$$
\mu\left(\mathfrak{S}_{Q(\varepsilon), \varepsilon^{1 / 4}}\right)>\rho_{1}
$$


where we denote

$$
\mathfrak{S}_{Q, \varepsilon}=\bigcup_{q<Q} \bigcup_{(a, q)=1} B\left(\frac{a}{q}, \varepsilon\right)
$$

Step 5. (conclusion).

Starting from (7) with $\varepsilon=\varepsilon_{0}$ small enough (depending on $\rho_{1}$ ), we perform again an iterative bootstrap (as in Step 3), invoking the following.

Lemma 8. Let $\mathfrak{S}_{Q, \varepsilon}$ be as above and let $n=n(\varepsilon) \in \mathbb{Z}_{+}$satisfying

$$
n<c \log \frac{1}{\varepsilon} \quad(c \text { depending on } \nu) \text {. }
$$

Assume

$$
\left(\nu^{(n)} * \mu\right)\left(\mathfrak{S}_{Q, \varepsilon}\right)=\sum \nu^{(n)}(g) \mu\left(g^{-1}\left(\mathfrak{S}_{Q, \varepsilon}\right)\right)>\kappa .
$$

Then we have

$$
\mu\left(\mathfrak{S}_{Q, \varepsilon^{\prime}}\right)>\kappa-e^{-c_{2} n}
$$

where

$$
\varepsilon^{\prime}=e^{-\frac{1}{4} \gamma n} \varepsilon
$$

The proof of Lemma 6 uses again Propositions 2 and 3.

Thus with $Q=Q\left(\varepsilon_{0}\right)$ fixed, $\varepsilon$ is gradually decreased and in the limit we obtain

$$
\mu\left(\left\{\frac{a}{q}: 1 \leq q<Q\left(\varepsilon_{0}\right), 0 \leq a_{1}, a_{2}<q\right\}\right)>\frac{1}{2} \rho_{1}>0 .
$$

This establishes Theorem B.

We conclude with some comments on the proof of Theorem C. For $m \geq 1$ we denote by

$$
\eta_{m}=\nu^{(m)} * \delta_{\theta}
$$

the measure on $\mathbb{T}^{2}$ ( $\delta_{x}$ stands here for the Dirac measure). It these notations, the assumption of Theorem $\mathrm{C}$ becomes

$$
\left|\hat{\eta}_{n}(b)\right|>\delta \quad \text { where } \quad b \in \mathbb{Z}^{2} \backslash\{0\} .
$$

The proof of steps (1)-(4) is quantitative, and even though $\eta_{m}$ is not $\nu$-stationary, these arguments can still be applied if one is willing to sacrifice a few powers of $\nu$.

For example, in step (1) we may conclude from (10) that for any $k<n$ there is some $N$ with $c_{3} k<\log N<c_{4} k$ and a set $\mathcal{F} \subset \mathbb{Z}^{2} \cap B(0, N)$ satisfying (a)-(c) 
of Lemma 1 for $\mu=\eta_{n-k}$ and $\beta=(\delta /\|b\|)^{C}$ (where $C$ and $c_{3}, c_{4}$, as well as all the other constants appearing below depend only on $\nu$ ). Similarly modifying steps (2)-(4) we conclude that for any $k^{\prime}$ in the range $C^{\prime} \log (\|b\| / \delta)<k^{\prime}<n$ there are $Q, \epsilon=Q^{-20}$ with $c_{3}^{\prime} k^{\prime}<\log Q<c_{4}^{\prime} k^{\prime}$ satisfying (cf. (7))

$$
\eta_{n-k^{\prime}}\left(\mathfrak{S}_{Q, \varepsilon}\right)>\left(\frac{\delta}{\|b\|}\right)^{C} \text {. }
$$

Let $n^{\prime}=n-k^{\prime}$ for $c_{5} \log (\|b\| / \delta)<k^{\prime}<n / 2$, with $c_{5}$ a large constant. Since $\eta_{n^{\prime}}=\nu^{\left(n^{\prime}\right)} * \delta_{\theta}$, if $c_{5}$ is sufficiently large, iteration of Lemma 6 imply that

$$
\delta_{\theta}\left(\mathfrak{S}_{Q, \varepsilon^{\prime}}\right)>\left(\frac{\delta}{\|b\|}\right)^{C}-\max \left(Q^{-c_{3}}, e^{-c_{2} n^{\prime}}\right)>0
$$

where

$$
\varepsilon^{\prime}<e^{-\frac{1}{4} \gamma n^{\prime}} \varepsilon<e^{-\frac{1}{8} \gamma n},
$$

i.e. $\theta \in \mathfrak{S}_{Q, \varepsilon^{\prime}}$. Since $Q<(\|b\| / \delta)^{C_{0}}$ for some $C_{0}$, equation (1) of Theorem C follows.

\section{REFERENCES}

[Be] D. Berend, Multi-invariant sets on tori, Trans. Amer. Math. Soc., 280 (2), 509-532.

[B-L] P. Bougerol, J. Lacroix, Products of random matrices with applications to Schrödinger operators, Birkhauser 1985.

[B] J. Bourgain, On the Erdös-Volkmann and Katz-Tao ring conjecture, GAFA, 13 (2003), 334-365.

[B-G] J. Bourgain, A. Gamburd, On the spectral gap for finitely-generated subgroups of $S U(2)$, preprint, submitted to Inventiones.

[E-L] M. Einsiedler, E. Lindenstrauss, Rigidity properties of $Z^{d}$-actions on tori and solenoids, Electron. Res. Announc. Amer. Math. Soc. 9 (2003), 99-110.

[F1] H. Furstenberg, Disjointness in ergodic theory, minimal sets, and a problem in Diophantine approximation, Math. Systems Theory, 1 (1967), 1-49.

[F2] H. Furstenberg, Stiffness of group actions, in 'Lie groups and ergodic theory', (Mumbai, 1996), 105-117, Tata Inst. Fund. Res. Stud. Math., 14, Tata Inst. Fund. Res., Bombay, 1998.

[G-M] I. Ya. Gol'dsheřd, G. A. Margulis, Lyapunov exponents of a product of random matrices. (Russian) Uspekhi Mat. Nauk 44 (1989), no. 5(269), 13-60; translation in Russian Math. Surveys 44 (1989), no. 5, 11-7.

[G] Y. Guivarc'h, private communication.

[G-S] Y. Guivarc'h, A.N. Starkov, Orbits of linear group actions, random walk on homogeneous spaces, and toral automorphisms, Ergodic Theory Dynam. Systems, 24 (2004), no. 3, 767-802.

[Ka-K] B. Kalinin, A. Katok, Invariant measures for actions of higher rank abelian groups, Smooth ergodic theory and its applications (Seattle, WA, 1999), 593-637, Proc. Sympos. Pure Math., 69, Providence, RI, 2001.

[K-S] A. Katok, R. Spatzier, Invariant measures for higher rank hyperbolic abelian actions, Ergodic Th. Dyn. Syst. 16 (1996), n 4, 751-778.

[K-T] N. Katz, T. Tao, Some connections between Falconer's distance set conjecture and sets of Furstenberg type, New York J. Math. 7 (2001), 149-187.

[M] G. Margulis, Problems and conjectures in rigidity theory, Mathematics: frontiers and perspectives, 161-174, Amer. Math. Soc., 2000. 
[Mu1] R. Muchnik, Orbits of Zariski dense semigroups of $\mathrm{SL}(n, \mathbb{Z})$, Ergodic Theory Dynam. Systems.

[Mu2] R. Muchnik, Semigroup actions on $\mathbb{T}^{n}$, Geometriae Dedicata, 110 (2005), 1-47.

[P] P. Pink, Strong approximation for Zariski dense subgroups over arbitrary global fields, Comment. Math. Helv. 75 (2000), no 4, 608-643.

[R] D. Rudolph, $\times 2$ and $\times 3$ invariant measures and entropy, Ergodic Theory Dynam. Systems 10 (1990), no. 2, 395-406.

[St] A.N. Starkov, Orbit closures of toral automorphism groups, preprint, Moscow, 1999.

[We] B. Weisfeiler, Strong approximation for Zariski-dense subgroups of semisimple algebraic groups, Ann. of Math. (2), 120 (1984), no. 2, 271-315. 